\section{Multiple Glassy States in a Simple Model System}

\author{
K. N. Pham, ${ }^{1}$ A. M. Puertas, ${ }^{1,2}$ J. Bergenholtz, ${ }^{3}$ S. U. Egelhaaf, ${ }^{1}$ \\ A. Moussaïd, ${ }^{1}$ P. N. Pusey, ${ }^{1}$ A. B. Schofield, ${ }^{1}$ M. E. Cates, ${ }^{1}$ \\ M. Fuchs, ${ }^{1}$ W. C. K. Poon ${ }^{1 *}$
}

Experiments, theory, and simulation were used to study glass formation in a simple model system composed of hard spheres with short-range attraction ("sticky hard spheres"). The experiments, using well-characterized colloids, revealed a reentrant glass transition line. Mode-coupling theory calculations and molecular dynamics simulations suggest that the reentrance is due to the existence of two qualitatively different glassy states: one dominated by repulsion (with structural arrest due to caging) and the other by attraction (with structural arrest due to bonding). This picture is consistent with a study of the particle dynamics in the colloid using dynamic light scattering.
Understanding the glass transition is an outstanding challenge for statistical and condensed-matter physics, with relevance throughout materials science as well as biology (1-3). In the multidisciplinary quest for understanding of glasses, the study of simple model systems occupies an important place. One of the simplest models amenable to theoretical study as well as experimentation is a collection of $N$ hard spheres of radius $R$ in volume $V$ at density (volume fraction) $\phi=(4 / 3) \pi R^{3} N / V$. Although there have been speculations about a hardsphere glass at least since Bernal (4), substantial progress began in the 1980 s with modecoupling theory (MCT) calculations (5) and experiments using colloids $(6,7)$. Further predictions from MCT have been substantially confirmed by colloid experiments and simulations (8), and novel features, such as spatially inhomogeneous particle dynamics, are still being revealed by new experimental probes (9). This close interplay between experiment, theory, and simulation has helped to give hard spheres the status of a reference system.

In a system of hard spheres, particles are increasingly caged by their neighbors as $\phi$ increases. At a critical density, $\phi_{g}$, this caging becomes effectively permanent, stopping all long-range particle motion, and the system can be considered nonergodic, or glassy. MCT captures the essential nonlinear feedback in this mechanism. Each particle is both caged and forms part of the cage of its neighbors. We present a combined experimental, theoretical, and simulational study of how the hard-sphere glass transition is perturbed by a short-range interparticle attraction ("stickiness"). We find

'Department of Physics and Astronomy, The University of Edinburgh, Mayfield Road, Edinburgh EH9 3JZ, UK. ${ }^{2}$ Department of Applied Physics, University of Almería, 04.120 Almería, Spain. ${ }^{3}$ Department of Chemistry, Physical Chemistry, Göteborg University, S-41296 Göteborg, Sweden.

*To whom correspondence should be addressed. Email:w.poon@ed.ac.uk that such an attraction first "melts" the hardsphere glass, and then a second, qualitatively different, glassy state is formed (Fig. 1). Sticky hard spheres therefore represent perhaps the simplest system in which multiple glassy states occur.

In our experiments, we used sterically stabilized polymethylmethacrylate (PMMA) particles (hard-sphere radius $R=202 \mathrm{~nm}$, polydispersity $=7 \%$ ) dispersed in cis-decalin. Computer simulations (10) predict that below $\phi=0.494$, the lowest free energy state is an ergodic fluid consisting of amorphously arranged particles exploring all available space. For $0.494<\phi<0.545$, fluid and crystal coexist. Above $\phi=0.545$, the system should fully crystallize. PMMA colloids follow this prediction closely, except that the homogeneous nucleation of colloidal crystals ceases at $\phi \approx 0.58(6)$, and this has been interpreted as a glass transition.

A measure of this arrest is provided by the dynamic structure factor $S(q, t)$, which quantifies the decay in time $(t)$ of density fluctuations with wavelength $2 \pi / q$. In an ergodic system, $S(q, t \rightarrow$ $\infty) \rightarrow 0$. The point at which $S(q, \infty)>0$ first occurs identifies the idealized glass transition. Dynamic light scattering confirms that in PMMA suspensions, a glass transition in this sense occurs at $\phi_{g} \approx 0.58$ (7). We induced a short-range attraction by adding a nonadsorbing polymer, polystyrene (radius of gyration $r_{\mathrm{g}}=17$ $\mathrm{nm})$. The polymer is excluded from the region between the surfaces of two nearby particles, leading to an excess osmotic pressure pushing the particles together. Thus, the particles experience an effective depletion attraction, well described (11) by the Asakura-Oosawa (AO) form (12), whose range and depth are controlled by the polymer's size and concentration, respectively. The dimensionless range of the depletion attraction can be estimated by $\xi=r_{\mathrm{g}} / R$ to be 0.08 .

Mixtures were homogenized by prolonged tumbling and then left undisturbed for observation. Because of the size of the particles, colloidal crystals can easily be detected by the naked eye as iridescent specks. The nonequilibrium behavior of this system at low densities, $\phi \leqslant$ 0.2 , has been studied before $(13,14)$; we show our observations at $\phi \geqslant 0.3$ (Fig. 1). According to theory (15), adding polymer at small $\xi$ expands the region of fluid-crystal coexistence, and this is what we observed (diamonds, Fig. 1). Again, consistent with theory, samples at higher $\phi$ were fully crystalline (inverted triangles). For samples with the highest polymer (16) and/or colloid concentrations, however, no crystallization was observed within a period of weeks to months (solid squares and circles), even though equilibrium statistical mechanics predicts either fluid-crystal coexistence (for the solid squares) or full crystallization (for the solid circles).

Consider a sample sequence (Fig. 1, from A to $\mathrm{E}$ ) at $\phi \approx 0.6$. In thermodynamic equilibrium, all of these samples should crystallize (15). The sample without any polymer was found to be a glass, consistent with $\phi_{\mathrm{g}} \approx$ 0.58 in pure hard spheres $(6,7)$. A sample with $\sim 1 \mathrm{mg} \mathrm{cm}^{-3}$ of polymer, however,

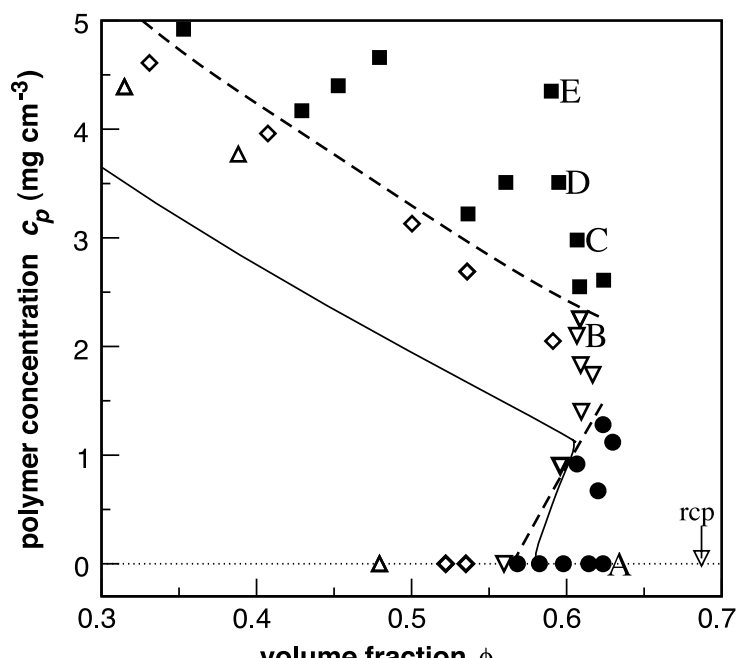

Konstanzer Online-Publikations-System (KOPS)

URL: http://www.ub.uni-konstanz.de/kops/volltexte/2007/3780/

Fig. 1. Equilibrium and nonequilibrium behavior of a colloid-polymer mixture at $\xi=0.08$. Samples that reached thermal equilibrium (open symbols) are as follows: fluid (triangles), fluid-crystal coexistence (diamonds), and fully crystalline (inverted triangles). Samples that did not reach thermal equilibrium (solid symbols) are as follows: repulsiondriven glass (circles) and attractiondriven glasses (squares). Dashed curves are guides to the eye of the observed glass transition lines. Solid curves are MCT predictions of glass transition lines $(17,18)$. Light-scattering data for samples labeled A through $\mathrm{E}$ are shown in Fig. 3. 
completely crystallized. This means that $\phi_{\mathrm{g}}$ had shifted to higher volume fractions. Suppression of crystallization was again observed at polymer concentration around 2.4 $\mathrm{mg} \mathrm{cm}{ }^{-3}$. From the behavior of all the samples, we find that the line of structural arrest at the high-density end of the phase diagram has a reentrant $(>)$ shape, which we then explored using MCT.

Given the interparticle potential, MCT predicts the glass transition using only the $(\phi$ dependent) equilibrium (or static) structure factor $S(q)$ as input, which was calculated by standard methods (17). The results shown in Fig. 1 correspond to an $\mathrm{AO}$ attraction with range $\xi=$ 0.08 . Given that there is essentially no adjustable parameter in the calculation (17), the agreement with data is remarkable (18).

Within the MCT framework, the reentrant glass transition is due to the existence of two kinds of glasses: one dominated by repulsion and the other by attraction (19). In the former, the key MCT modes giving rise to structural arrest are those with a wavelength close to the average interparticle spacing, corresponding to particles being caged by their neighbors. Turning on a short-range attraction leads to clustering of the particles forming the cage and opens up holes (free volume), ultimately melting the glass. Increasing the attraction further, however, eventually leads to another structural arrest, in which the key MCT modes are now those with wavelengths comparable to the range of the interparticle attraction, suggesting that particles stick to their neighbors with long-lived bonds. These two kinds of arrest may be termed repulsion- and attraction-driven glasses, respectively.

To confirm this interpretation, we simulated a system of 1000 particles interacting throgh a steep repulsion $\left(\propto r^{-36}\right)$ and an AO attraction with $\xi=0.10$. To prevent crystallization and gas-liquid phase separation, a small polydispersity in particle size and a weak repulsive barrier beyond the AO attraction were introduced (20). In this system without any attraction (but retaining the barrier), we find a (repulsion-driven) glass tran-

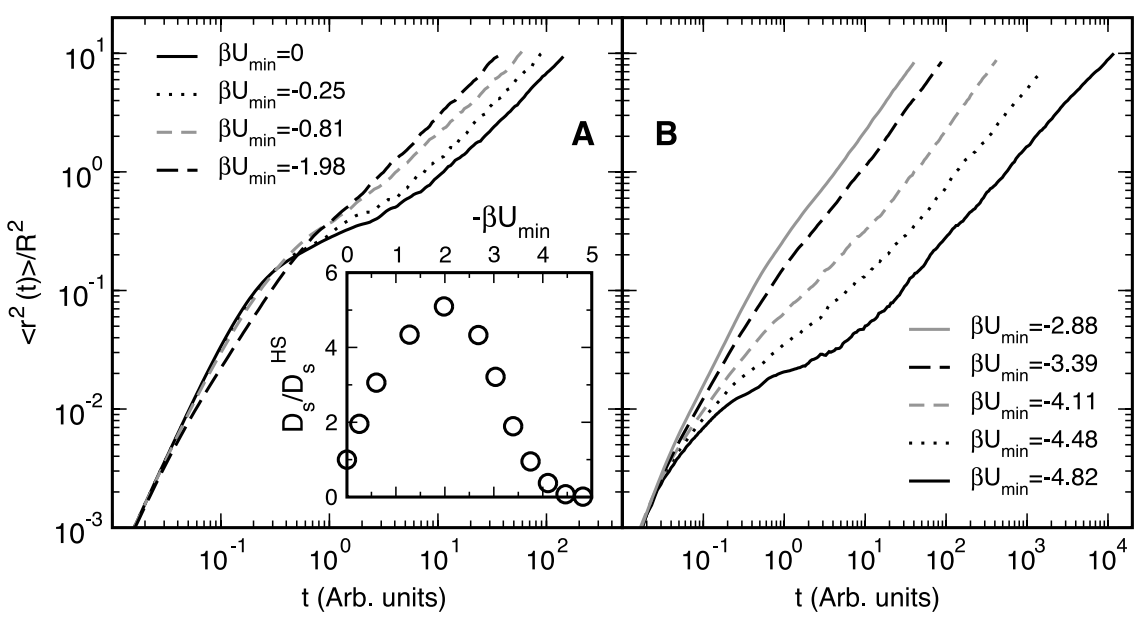

Fig. 2. Plots of mean squared displacements $\left\langle r^{2}(t)\right\rangle$ in units of particle radius squared as a function of time, $t$, from simulations of slightly polydisperse, almost-hard spheres with $\mathrm{AO}$ attraction and a weak repulsive barrier $(20)$ at $\phi=0.50$, for increasing strengths of attraction, $\left(U_{\text {min }}\right)$ as labeled [with the inverse thermal energy $\beta=\left(k_{B} T\right)^{-1}$ ], (A) for $0 \geq \beta U_{\min } \geq-1.98$ and $(\mathbf{B})$ for $-2.88 \geq$ $\beta U_{\min } \geq-4.82$. The inset shows the dependence of the single-particle long-time diffusion coefficient $\left(D_{\mathrm{s}}\right.$ in units of the hard-sphere value $\left.D_{\mathrm{S}}{ }^{\mathrm{HS}}\right)$ on attraction strength.

Fig. 3. Normalized collective dynamic structure factors $f(q, t)$ obtained from light scattering at $q R=2.93$ as function of time, $t$, for samples A through E (Fig. 1). The inset replots the data with an expanded vertical axis; in particular, the decay in sample $C$ is consistent with linearity in log time for over a decade in time (25).

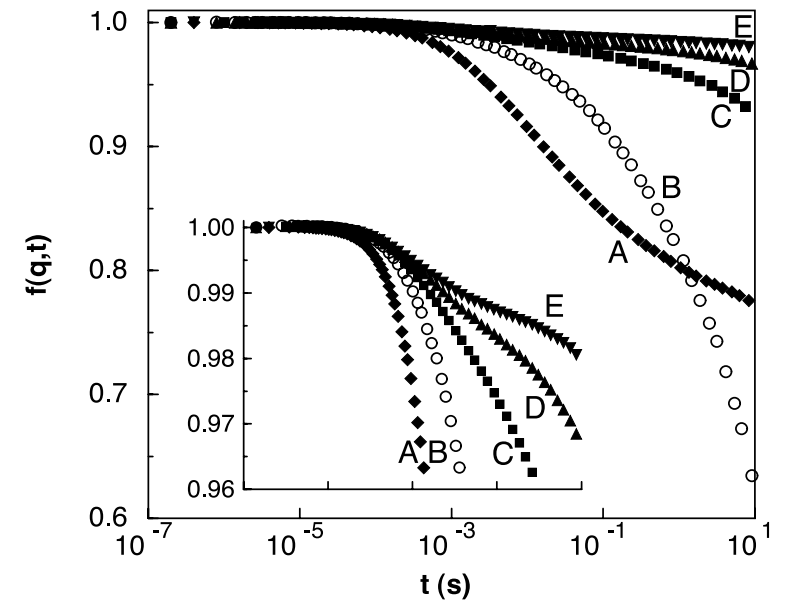

sition at $\phi_{\mathrm{g}} \sim 0.55$. Figure 2 shows the mean squared displacement, $\left\langle r^{2}(t)\right\rangle$, as we progressively turned on a short-range attraction at $\phi=0.50$.

With no attraction, $\left\langle r^{2}(t)\right\rangle$ shows signs of a plateau (slowing down) at $\sim 10^{-1} R^{2}$ due to the proximity of the glass transition at $\phi_{\mathrm{g}} \sim$ 0.55 . The length scale at which the slowing down takes place is connected to the socalled Lindemann length over which a particle explores its cage, which becomes permanent at $\phi_{\mathrm{g}}(5,8)$. The plateau gradually disappears as the short-range attraction is increased; concomitantly, the long-time diffusion coefficient $\left(\propto d\left\langle r^{2}(t)\right\rangle / d t\right.$ at long times) increases. This suggests that increasing the attraction at constant density takes us further away from the glass transition, which is consistent with the repulsion-driven glass transition line on Fig. 1 slanting to the right. Increasing the attraction further, however, introduces another incipient plateau into $\left\langle r^{2}(t)\right\rangle$, suggesting that the system is again coming close to a glass boundary. This plateau occurs at $\left\langle r^{2}\right\rangle \sim 10^{-2} R^{2} \sim \xi^{2}$. The particles become progressively more trapped in potential wells, until quasi-permanent bonds are formed at the attraction-driven glass transition.

Besides giving the positions of the reentrant glass transition line, MCT also makes definite predictions about the form of $S(q, t)(19)$; in particular, that $S^{(\mathrm{rep})}(q, \infty)$ is significantly smaller than $S^{\text {(att) }}(q, \infty)$, reflecting the weaker localization by the cage (rep, repulsion) than by interparticle bonds (att, attraction). The correlations in the intensity of singly scattered laser light from a suspension gives the normalized dynamic structure factor $f(q, t)=S(q, t) / S(q)$. Because our samples are somewhat turbid, we used two-color dynamic light scattering to access the single-scattering intensity information, and therefore $f(q, t)(21)$. Data collected at $q R=$ 2.93, corresponding to spatial correlations slightly longer than the average particle distance, are shown in Fig. 3, for samples A through E in Fig. 1.

Our data for sample A are consistent with previous results $(22,23)$. The point of inflection at $t \sim 0.1 \mathrm{~s}$ is indicative of the glassy plateau, $f(q, \infty) \sim 0.7$, that lies outside our time window. The curve for sample B has no inflection point, because a weak short-range attraction has melted the glass into a (metastable) ergodic fluid (which will eventually crystallize). A stronger attraction brings a dramatic change. The curve for sample $\mathrm{C}$ decays by $\leqslant 7 \%$ in the time window shown, whereas the next two curves (for samples D and E) hardly decay at all (inset, Fig. 3). These results are consistent with the MCT prediction that $S^{(\text {rep })}(q, \infty)$ is significantly smaller than $S^{\text {(att) }}(q, \infty)(19,24)$.

The existence of multiple glassy states opens the possibility of glass-to-glass transitions and to 
the appearance of endpoints on glass transitions lines where the difference between two glasses vanishes (19). Close to such points, the structural relaxation will exhibit an especially broad distribution of relaxation times, because the system anticipates arrest in either of two glassy structures. Sample C (inset, Fig. 3) displays rather anomalous, stretched-out dynamics over an appreciable range in time. The functional form of this dynamics is consistent with MCT predictions (25) as well as simulations (26).

A model hard-sphere colloid with a short-range attraction induced by added polymer shows a reentrant glass transition at high densities. This system is sufficiently well characterized that we were able to compare our observations with MCT calculations with no adjustable parameters. These calculations, molecular dynamics simulations, and light-scattering experiments all suggest that the reentrance is due to the existence of two qualitatively distinct kinds of glasses, dominated by repulsion and attraction, respectively (27). Sticky hard spheres are also predicted to show an isostructural crystal-crystal transition (28). If the stickiness is modeled by a square well, this transition vanishes when the well width is between 6 and $7 \%$ of the hard core (28), whereas the glass-glass transition disappears at $\sim 5 \%$ attraction width (19). The similarity is striking: The same mechanism, a competition between attraction and repulsion, underlies both phenomena.

\section{References and Notes}

1. See the wide-ranging survey articles in Science 267, $1887(1995)$ and particularly $(2,3)$

2. C. A. Angell, Science 267, 1924 (1995).

3. F. H. Stillinger, Science 267, 1935 (1995).

4. J. D. Bernal, Proc. R. Soc. London Ser. A 280, 299 (1964).

5. U. Bengtzelius, W. Götze, A. Sjölander, J. Phys. C 17, 5915 (1984)

6. P. N. Pusey, W. van Megen, Nature 320, 340 (1986).

7. Phys. Rev. Lett. 59, 2083 (1987).

8. See the review and references cited in W. Götze, J. Phys. Condens. Matter 11, A1 (1999).

9. E. R. Weeks, J. C. Crocker, A. C. Levitt, A. Schofield, D. A. Weitz, Science 287, 627 (2000).

10. W. G. Hoover, F. H. Ree, J. Chem. Phys. 49, 3609 (1968).

11. D. Rudhardt, C. Bechinger, P. Leiderer, Phys. Rev. Lett. 81, 1330 (1998).

12. S. Asakura, F. Oosawa, J. Chem. Phys. 22, 1255 (1954).

13. W. C. K. Poon, A. D. Pirie, P. N. Pusey, Faraday Discuss. 101, 65 (1995).

14. W. C. K. Poon et al., Faraday Discuss. 112, 143 (1999).

15. H. N. W. Lekkerkerker et al., Europhys. Lett. 20, 559 (1992).

16. Even at the highest polymer concentrations reached in this work, we are still considerably below the overlap concentration $c^{*} \sim 30 \mathrm{mg} \mathrm{cm}^{-3}$, where the effective coil volume fraction is $\sim 1$.

17. We calculated $S(q)$ for hard spheres with an attractive Yukawa tail in the mean spherical approximation and exploited a mapping to the $\mathrm{AO}$ attraction tail. State points are paired up for $\phi \rightarrow 0$ by equating the large- $q$ behavior of the Mayer functions (amplitude $A$ and period $\xi^{\prime}$ of oscillation) in the limits $\xi \rightarrow 0$ and for strong attractions. $A$ is connected to the polymer concentration and $\xi^{\prime}$ to the polymer size. The ade- quacy of this mapping has been confirmed by Monte Carlo simulations at a number of $\mathrm{AO}$ state points. The dominant uncertainty when comparing the theory and simulations to the experiments arises from the unknown many-body effects not captured in the $\mathrm{AO}$ potential. We expect that $\mathrm{AO}$ overestimates the effective strength of the attraction. MCT predicts $\phi_{\mathrm{g}}=0.52$ for hard spheres (HS); we apply a scaling factor $0.58 / 0.52$ to agree with the experimental value of $\phi_{\mathrm{g}}^{\mathrm{HS}}=0.58$.

18. The MCT ideal-glass criterion is when $S(q, t \rightarrow \infty)>0$. In Fig. 1 we show where crystallization was not observed on the week/month time scale, which is at least indicative of a nearby glass transition.

19. K. Dawson et al., Phys. Rev. E 63, 011401 (2000) and references therein.

20. A flat top-hat distribution of particle size with polydispersity $10 \%$ was chosen, and a smooth repulsive potential barrier of maximal height $1 k_{\mathrm{B}} T$ (the thermal energy) and width $2 R(1-\xi)$ modeled by a quartic polynomial which gives continuous total force was added. For computational efficiency and because we are interested in the long-time structural relaxation only, Newtonian (rather than Brownian) dynamics was used.

21. P. N. Segrè et al., J. Mod. Opt. 42, 1929 (1995).

22. W. van Megen, S. M. Underwood, Phys. Rev. E 49, 4206 (1994).

23. Direct comparison with (22) is complicated by differing polydispersities, which lead to differing randomclose-packing densities, $\phi_{\text {rcp }}$ : The particle dynamics is a very sensitive function of $f=\left(\phi_{\mathrm{rcp}}-\phi\right) / \phi_{\mathrm{rcp}}$ near $\phi_{\text {rcp. }}$. We measured $\phi_{\text {rcp }} \sim 0.68$ for our particles. Our data for sample $A$, which has $f=0.1$, are consistent with what van Megen and Underwood showed at comparable wavevectors for a sample with $\phi=$ 0.578 (see their figure 6a). They took $\phi_{\text {rcp }}=0.64$, so that $\phi=0.578$ corresponds to $f=0.097$.
24. The $f(q, t)$ data (Fig. 3$)$ indicate the freezing-in of large collective density fluctuations, whereas the $\left\langle r^{2}(t)\right\rangle$ data (Fig. 2) suggest strongly localized singleparticle motion.

25. The scattering function for sample $C$ is compatible with logarithmic decay, $S(q, t)=F(q)-H(q) \log (t)$ $\left.t_{*}\right)$, over more than a decade in time, as predicted by MCT for state points close to where the glass-glass transition should vanish (19). Such dynamics have been observed before in less well-characterized systems $(29,30)$.

26. A. M. Puertas, M. Fuchs, M. E. Cates, Phys. Rev. Lett. 88, 098301 (2002).

27. The existence of multiple glassy states in a single material has been suggested in atomic systems [such as $\mathrm{SiO}_{2}$ and $\mathrm{GeO}_{2}$ (31) and water (32)]. The underlying physics in these network formers (33), however, is almost certainly distinct from that we have reported here for the simple model system of sticky hard spheres.

28. P. Bolhuis, M. Hagen, D. Frenkel, Phys. Rev. E 50, 4880 (1994).

29. F. Mallamace et al., Phys. Rev. Lett. 84, 5431 (2000).

30. E. Bartsch, M. Antonietti, W. Schupp, H. Sillescu, J. Chem. Phys. 97, 3950 (1992).

31. O. B. Tsiok et al., Phys. Rev. Lett. 80, 999 (1998).

32. O. Mishima, Y. Suzuki, J. Chem. Phys. 115, 4199 (2001).

33. G. Franzese et al., Nature 409, 692 (2001).

34. K.N.P. holds a UK Overseas Research Studentship and is partially supported by the University of Edinburgh. A.M.P., J.B., and M.F. were funded by the Ministerio de Educación y Cultura, Spain; the Swedish National Science Research Council; and the Deutsche Forschungsgemeinschaft (grant Fu 309/3), respectively.

20 November 2001; accepted 22 February 2002 